\title{
Barrier protective effects of 2,4,6-trihydroxy-3-geranyl acetophenone on lipopolysaccharides-stimulated inflammatory responses in human umbilical vein endothelial cells
}

\begin{abstract}
Pharmocological relevance: 2,4,6-trihydroxy-3-geranyl acetophenone (tHGA), is a phloroglucinol compound found naturally in Melicope ptelefolia. Melicope ptelefolia has been used traditionally for centuries as natural remedy for wound infections and inflammatory diseases.

Aim of the study: Endothelial barrier dysfunction is a pathological hallmark of many diseases and can be caused by lipopolysaccharides (LPS) stimulation. Therefore, this study aims to investigate the possible barrier protective effects of tHGA upon LPS-stimulated inflammatory responses in human umbilical vein endothelial cells (HUVECs).

Materials and methods: HUVECs were pretreated with tHGA prior to LPS stimulation, where inflammatory parameters including permeability, monocyte adhesion and migration, and release of pro-inflammatory mediators were examined. Additionally, the effect of tHGA on F-actin rearrangement and adhesion protein expression of LPS-stimulated HUVECs was evaluated.

Results: It was found that pretreatment with tHGA inhibited monocyte adhesion and transendothelial migration, reduced endothelial hyperpermeability and secretion of prostaglandin E2 (PGE2). Additionally, tHGA inhibited cytoskeletal rearrangement and adhesion protein expression on LPS-stimulated HUVECs.

Conclusion: As the regulation of endothelial barrier dysfunction can be one of the therapeutic strategies to improve the outcome of inflammation, tHGA may be able to preserve vascular barrier integrity of endothelial cells following LPS-stimulated dysfunction, thereby endorsing its potential usefulness in vascular inflammatory diseases.
\end{abstract}

Keyword: 2,4,6-trihydroxy-3-geranyl acetophenone (tHGA); Melicope ptelefolia; Phloroglucinol; Barrier protective effect; Endothelial dysfunction; Lipopolysaccharides 